山्山FFRANÇAISE

$>\mathrm{DE}$

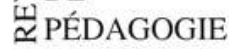

\section{Revue française de pédagogie}

Recherches en éducation

177 | octobre -décembre 2011

La politique ZEP en France, laboratoire des politiques

d'éducation?

\title{
Les ZEP, miroir grossissant des évolutions et contradictions du système éducatif français
}

ZEP or the magnifying mirror of the developments and contradictions of the French educational system

Las ZEP, espejo de aumento de las evoluciones y contradicciones del sistema educativo francés

Die ZEP als Vergrößerungsspiegel der Entwicklungen und Widersprüche des französischen Schulsystems

\section{Marceline Laparra}

\section{OpenEdition}

Édition électronique

URL : http://journals.openedition.org/rfp/3395

DOI : $10.4000 /$ rfp.3395

ISSN : 2105-2913

Éditeur

ENS Éditions

Édition imprimée

Date de publication : 15 décembre 2011

Pagination : 47-60

ISBN : 978-2-84788-353-4

ISSN : 0556-7807

Référence électronique

Marceline Laparra, «Les ZEP, miroir grossissant des évolutions et contradictions du système éducatif français », Revue française de pédagogie [En ligne], 177 | octobre -décembre 2011, mis en ligne le 15 décembre 2015, consulté le 19 avril 2019. URL : http://journals.openedition.org/rfp/3395 ; DOI : 10.4000/rfp.3395 


\title{
Les ZEP, miroir grossissant des évolutions et contradictions du système éducatif français
}

\author{
Marceline Laparra
}

La création des ZEP en France peut être considérée comme une tentative de résoudre - dans un contexte de généralisation de l'accès à l'enseignement secondaire - la contradiction entre la nécessité républicaine de proposer le même enseignement à tous et l'exigence démocratique de prendre en compte les différences et inégalités socioculturelles entre les élèves. Les modalités de leur mise en œuvre et la multiplication des objectifs assignés à l'institution scolaire ont toutefois laissé aux enseignants la charge de concilier des finalités et objectifs hétérogènes, voire contradictoires. Peu instrumentés pour cela, ceux-ci n'ont pu y parvenir qu'en "diversifiant » les outils - manuels, fichiers, recueils d'exercices, etc. - qu'ils utilisent, les situations ou modes d'organisation du travail en classe qu'ils mettent en œuvre. Nombre de travaux permettent néanmoins de penser que cet éclectisme pédagogique, s'il n'est pas de nature ni d'ampleur différente en ZEP ou hors ZEP, n'y a pas les mêmes effets, et qu'il contribue à une "dé-didactisation » des problèmes, source de difficultés et d'inégalités d'apprentissage, qui semble particulièrement défavorable aux élèves de milieux populaires ou issus des familles et des quartiers les plus précarisés. Ce qui amène à se demander s'il ne faut pas procéder au contraire à une forte didactisation des problèmes rencontrés par les élèves dans leur scolarité et mettre en question les processus dominants d'adaptation des enseignements à ce qu'on pense être les particularités des élèves.

Mots-clés (TESE) : démocratisation de l'enseignement, difficulté d'apprentissage, zone d'éducation prioritaire, pratique pédagogique, politique en matière d'éducation.

Les considérations que l'on va lire ne sont pas le fruit d'un travail de recherche à proprement parler mais plutôt des réflexions de quelqu'un qui à la fois a été un acteur et un observateur des politiques d'éducation prioritaire en France. Le défi que pose à la société française l'existence d'un échec scolaire important dans les classes sociales les moins favorisées économiquement et culturellement est très souvent rapproché de celui que connaissent les systèmes éducatifs d'autres pays développés (Ministère de l'Éducation nationale, 2003). Il comporte néanmoins une très forte spécificité qui s'explique par l'histoire de l'école républicaine et par la manière dont elle a résolu les conflits entre les intérêts potentiellement divergents des différents groupes sociaux. Jusqu'aux années soixante et soixante-dix, sous couvert d'un enseignement iden- 
tique pour tous, l'école française s'est employée - à la satisfaction quasi générale - à dégager deux types d'élites, l'une au sein des populations rurales, l'autre au sein des classes urbaines favorisées. Ce consensus a explosé avec la massification de l'enseignement secondaire et le débat s'est alors cristallisé sur deux questions : quelle place doit-on accorder respectivement aux deux missions de l'école, l'éducation et l'enseignement, et peut-on dispenser le même enseignement à tous les élèves, quelle que soit leur origine sociale ? Les réponses à ces questions n'ont jamais été claires et les politiques éducatives qui se sont enchaînées ont pu alors poursuivre des objectifs différents, tout en disant se fonder sur les mêmes valeurs. Par exemple, les politiques d'inspiration libérale de la dernière décennie se réclament de ce qui fit la réussite de l'école de Jules Ferry.

Se sont alors empilées des mesures empruntant à des logiques très différentes : réécriture incessante des programmes $^{1}$, création des zones d'éducation prioritaires (ZEP) et changements dans leur mode de pilotage, succession de dispositifs nationaux d'évaluation, modifications dans l'organisation de la formation des maîtres (des mesures De Peretti à la mastérisation en passant par la création puis la suppression des IUFM)... Cet entassement de réformes de plus ou moins grande portée a produit la plus grande confusion sur le plan des contenus à enseigner et des pédagogies à mettre en œuvre. Les recherches sur l'école n'ont pu que refléter ces changements d'orientation. Les enseignants se sont retrouvés seuls, chacun dans sa classe, à devoir essayer de répondre aux injonctions contradictoires que comportaient inévitablement toutes ces mesures, aussi bien sur le plan de l'organisation de leur classe que du tri à effectuer parmi des matériels pédagogiques surabondants ou du choix à opérer entre des modèles pédagogiques antagonistes. Ils l'ont fait au hasard des spécificités de l'école dans laquelle ils travaillaient, de celles de leur formation disciplinaire et professionnelle et des particularités des élèves qui leur étaient confiés. Ils se sont tous retrouvés de ce fait en situation d'insécurité professionnelle, mais celle-ci ne pouvait être que plus forte et dommageable pour ceux qui avaient en charge les élèves les plus en difficulté. Le débat national sans cesse recommencé et jamais abouti sur ce que doivent être les missions de l'école a ainsi occulté depuis quarante ans la réalité de ce qui se passe dans les écoles au quotidien et des transformations qui s'y jouent, rendant de ce fait très difficile l'analyse des processus effectifs de production de l'échec au sein de l'institution scolaire.

\section{DU CONSENSUS À L'ÉLABORATION SANS CESSE REMANIÉE D'UNE SOLUTION}

\section{Du consensus au recours aux ZEP}

Dès les années 1880, l'école républicaine s'est trouvée écartelée entre, d'une part, des valeurs universalistes qui semblaient lui imposer de traiter de manière uniforme tous les élèves qui lui étaient confiés et, d'autre part, les intérêts divergents en matière de scolarisation des groupes sociaux majoritaires sur le plan démographique et des groupes sociaux dominants sur le plan économique et culturel, qui ne pouvaient qu'entraîner une segmentation du système éducatif en filières différentes. Rappelons ici que ce ne sont pas les seules classes favorisées qui ont trouvé leur compte dans l'étanchéité existant entre un enseignement court pour le plus grand nombre et un enseignement long pour une très petite minorité. Eugen Weber (1983) a montré que le monde rural n'attendait pas, au début du $x x^{e}$ siècle, que l'école conduise tous les élèves au certificat d'études, ce qui n'a jamais, et de loin, été le cas, mais qu'elle dégage en son sein une élite restreinte, les élèves ainsi sélectionnés pouvant bénéficier individuellement d'une promotion sociale utile non seulement à eux-mêmes mais à leur famille. La partition du système éducatif en deux filières, qui permettait de dégager à deux niveaux différents deux types d'élite, a été alors l'objet d'un large consensus qui peu ou prou a perduré, au prix d'aménagements successifs, jusqu'aux années cinquante, et a réussi à concilier des objectifs apparemment contradictoires.

Tant que l'échec scolaire ne valait pas exclusion du monde du travail pour les populations concernées, le consensus a perduré et l'amélioration d'un système profondément inégalitaire n'a pas été au premier plan des préoccupations collectives, ce qui explique par exemple que le Front populaire n'ait que peu agi en la matière (il s'est surtout attaché au développement de l'éducation populaire en dehors de l'école). II faut attendre que la population devienne majoritairement urbaine pour que soit allongée la scolarité obligatoire et que soit progressivement créé le collège unique au cours des années soixante. L'échec scolaire devient rapidement un enjeu social important (Isambert-Jamati, 1985 ; CRESAS, 1981), le collège unique montrant très vite son incapacité à faire réussir tous les élèves également. Les tensions entre le respect des valeurs universalistes demandant un enseignement identique pour tous et la nécessaire prise en compte des différences socioculturelles ne trouvent plus matière à se résorber dans un compromis, que celui-ci respecte les termes de l'ancien compromis ou qu'il en trouve de nouveaux : 
dégager des élites différentes au sein de différents groupes sociaux n'est plus une solution viable ${ }^{2}$. S'impose l'exigence de faire réussir les élèves de milieux peu favorisés aussi bien que les autres. La création des ZEP est une tentative pour y parvenir. Le fait qu'on ait dû procéder à plusieurs relances et qu'on ait augmenté très fortement le nombre d'élèves en bénéficiant (Kherroubi \& Rochex, 2002 ; Rochex, 2008) doit être considéré moins comme le signe d'un pilotage déficient que comme la résultante d'un triple phénomène :

- l'impossibilité de modifier les termes du problème (unicité et différenciation du système) en privilégiant une exigence au détriment de l'autre ;

- la persistance de l'échec scolaire, socialement déterminé, qui tend de plus en plus à être considéré comme un problème voire comme un scandale public (Ravon, 2000) ;

- l'absence de solution de rechange.

En d'autres termes, on est contraint de poursuivre une politique qui a montré ses limites, faute d'en avoir de meilleure. On ne peut qu'essayer d'en modifier les contours, lui assigner de nouveaux objectifs ou en changer les modes de gestion.

\section{Les variations dans la réponse apportée par les ZEP}

La réponse quantitative est la plus facile à mettre en œuvre (elle a prévalu en un premier temps) et correspond à une demande forte des acteurs de terrain. Diminuer le nombre d'élèves par classe, mettre à disposition des maîtres en surnombre dans les écoles, attribuer des primes aux enseignants et financer le budget de fonctionnement de projets innovants semblent être des mesures de bon sens : les élèves en grande difficulté ne peuvent qu'avoir plus de chances de réussir dans des classes moins chargées avec des enseignants mieux payés. Mais les limites d'une telle politique sont vite apparues, les résultats dans la lutte contre l'échec scolaire n'étant pas souvent à la hauteur des attentes.

La réponse qualitative a pris des aspects variés mais n'a que rarement porté sur les situations d'apprentissage mises en œuvre dans les classes, malgré les injonctions émanant des responsables ministériels de se recentrer sur les apprentissages ${ }^{3}$. On a cherché à améliorer les relations de l'école avec son environnement, tout particulièrement avec les parents et les associations, à mobiliser les ressources locales en créant des passerelles avec les médiathèques, les centres sociaux et culturels (le lien avec les ressources locales devant constituer une large part du travail des coordinateurs dont on a doté les ZEP). À l'intérieur de l'école, on a prôné le travail en équipe et le travail en projet aussi bien à l'échelle de la classe qu'à celle de l'école. On a favorisé le développement de dispositifs d'aide, notamment d'aide aux devoirs, aussi bien dans le cadre scolaire que dans le périscolaire. Les enseignants à l'initiative de tels projets se réclamaient souvent de mouvements pédagogiques (comme l'institut coopératif de l'école moderne qui promeut la pédagogie Freinet, le groupe français d'éducation nouvelle, ou encore le cercle de recherche et d'action pédagogique qui édite les Cahiers pédagogiques, etc.) ou de recherches-actions menées dans des didactiques comme celles du français ou des mathématiques ${ }^{4}$. Tous les enseignants de ZEP disent chercher à ce que leurs élèves donnent du sens au travail scolaire. À plusieurs reprises, le ministère a cherché à capitaliser les innovations pédagogiques produites par les équipes de terrain aux fins d'en assurer une large diffusion. Mais il s'est alors contenté de procéder à une recension d'activités extrêmement diverses sans organiser une véritable réflexion collective sur les objectifs ou la portée de ces innovations. Certaines ont été sélectionnées par l'institution à des fins modélisantes (les enseignants sont censés les reproduire dans leur classe), sans que soit précisé selon quels critères elles avaient été retenues.

Les ZEP se sont vu assigner des objectifs différents au cours de leur histoire ${ }^{5}$. Cela témoigne bien sûr des changements d'inspiration dans les politiques nationales, mais cela renvoie aussi à la difficulté de faire évoluer le système éducatif autrement que par à-coups, sans que soient évalués les effets de recommandations successives. En un premier temps, les enseignants doivent lutter contre les mécanismes d'exclusion, l'institution incitant à bannir les redoublements, à organiser l'enseignement en cycles, à ménager des passerelles entre les différents niveaux (grande section de maternelle et $\mathrm{CP}, \mathrm{CM} 2$ et sixième, troisième et seconde). Ces mesures ne sont pas particulières aux ZEP mais elles occupent une place très importante dans les projets des établissements de l'éducation prioritaire. En un deuxième temps, les ZEP doivent développer une politique d'excellence ${ }^{6}$, en important dans les écoles et les collèges les dispositifs réservés aux établissements élitistes (comme les classes bilingues ou les classes musicales), ou en permettant à leurs meilleurs élèves d'accéder aux filières les plus réputées, comme les classes préparatoires aux grandes écoles, grâce à la création d'écoles d'été ou d'années propédeutiques. Elles doivent enfin identifier les besoins particuliers de leurs élèves pour que cha- 
cun d'entre eux soit en situation de réussite, quitte à ce que ce soit dans des parcours différenciés.

Du fait de leur succession très rapide, ces objectifs différents finissent par se cumuler, les enseignants restant libres, chacun dans sa classe, de répondre à l'un ou l'autre, ou à tous à la fois, à charge pour eux de les rendre compatibles et de les articuler entre eux. Mais ils peuvent surtout les trouver trop éloignés de leurs problèmes et peu opératoires pour inspirer les microchoix que leur impose le quotidien de la classe. Une telle situation ne peut conduire qu'à un sentiment d'abandon dont les effets sont obligatoirement négatifs sur le travail qu'ils mènent avec leurs élèves.

\section{ENTRE PILOTAGE NATIONAL FORT ET ANOMIE GÉNÉRALISÉE}

\section{De l'élève générique des textes réglementaires aux élèves réels}

Comme tous les autres enseignants français, les enseignants de ZEP sont livrés à eux-mêmes pour interpréter les consignes nationales en fonction des particularités de leurs élèves. Sans doute parce qu'elle se pose en garante des valeurs universalistes, l'institution s'est toujours interdit de différencier les contenus d'enseignement selon les filières. On retrouve d'une filière à une autre les mêmes contenus, le travail d'adaptation de ceux-ci à la spécificité de chaque filière restant à la charge des enseignants. Chaque renouvellement de programme voit se reproduire le même phénomène. En voici deux exemples pris à deux époques différentes et concernant des publics très distincts :

- Avant la guerre de 1914-1918, les programmes d'histoire appliqués dans les sixièmes et cinquièmes des lycées et en première année de brevet supérieur sont très proches les uns des autres, mais les élèves n'ont pas le même âge et n'obtiennent pas à terme le même diplôme. Les enseignants des classes de brevet disposent de deux fois moins de temps que ceux de sixième et cinquième. À eux de décliner les mêmes contenus (par exemple l'Égypte antique) en tenant compte de ces contraintes. Si on compare les manuels écrits par un même auteur pour ces deux types de classe, on s'aperçoit que celui-ci opère dans le manuel destiné aux élèves préparant le brevet une simple condensation de ce qui a été écrit dans le manuel destiné aux élèves de sixième (Laparra, 1991).
- Quant aux programmes actuels de français des classes des sections d'enseignement général et professionnel adapté (SEGPA, voir MEN, 1998), ils demandent aux enseignants d'initier leurs élèves aux mêmes notions que celles qui sont présentées aux élèves de sixième et cinquième, en les « adaptant » aux possibilités de leurs élèves.

II n'y aura, durant toute l'histoire des ZEP, aucune instruction pour spécifier les programmes. Les enseignants doivent les adapter aux réalités de terrain, tout en étant censés les respecter à la lettre. Comme dans le reste du système éducatif, ils doivent doter tous les élèves de tous les outils intellectuels et culturels leur permettant d'avoir la maîtrise du monde dans lequel ils seront appelés à vivre à leur sortie de l'école. Cet objectif est celui qui leur est assigné, année après année, quelles que soient les conceptions qu'ont les rédacteurs des programmes de ce qu'il est nécessaire d'enseigner à tous. Aux enseignants de le respecter, en tenant compte des particularités de leur lieu d'exercice. Une telle " adaptation » d'objectifs aussi généraux ne va déjà pas de soi quand il y a consensus sur les contenus à enseigner ; elle devient extrêmement problématique quand un tel consensus n'existe plus. Or le renouvellement très rapide des programmes nationaux depuis les années quatre-vingt prouve à lui seul que c'est le cas : tour à tour, on doit se centrer sur les " apprentissages fondamentaux ", souvent ramenés $\mathrm{au}$ " lire, écrire, compter ", doter les élèves de savoirs, de savoir-faire et de savoir-être, ou construire un " socle commun de compétences " qui laisse une telle marge à l'interprétation (que veut dire par exemple "savoir communiquer " ou " savoir retrouver des informations dans un texte " ?) qu'on a pu aussi bien reprocher à l'école de poursuivre une ambition démesurée qu'y voir une volonté de réduire a minima les savoirs prescrits.

On retrouve aggravée une constante du système éducatif français : la liberté qu'ont dans le choix de leurs méthodes pédagogiques les enseignants, jointe à l'absence de toute procédure d'agrément du matériel pédagogique (à la différence de ce qui se passe dans certains pays voisins), a toujours laissé à ceux-ci le soin de décliner des programmes qui sont écrits en fonction d'un élève abstrait, en quelque sorte générique. Ils doivent opérer la transformation de cet élève "générique " en des élèves "spécifiques " pour tenir compte, dans la mise en œuvre des programmes, des particularités socioculturelles de leurs élèves, de leur niveau, voire de leurs « besoins ». Cette opération présente déjà des difficultés quand les élèves 
appartiennent aux classes moyennes et aisées qui ont fourni la figure de l'élève générique. Elle devient redoutable quand les élèves réels que les enseignants ont devant eux ne correspondent guère à cet élève théorique et qu'ils n'ont souvent d'autre ressource que d'opérer des choix au coup par coup, au fil de leur activité, en surestimant comme en sous-estimant les capacités de leurs élèves, ce qui ne peut que nuire à l'efficacité de ce qu'ils font. Les contradictions que l'institution n'a pas résolues ne sauraient l'être magiquement par chacun d'entre eux.

L'institution perçoit bien la situation d'impossibilité professionnelle dans laquelle les enseignants les plus exposés se retrouvent et qui tient tout autant à cette fausse liberté qu'aux problèmes de discipline posés par les élèves. Elle croit alors leur venir en aide en multipliant les dispositifs d'évaluation et en les invitant à en exploiter les résultats (c'est ainsi par exemple qu'ont été légitimées, à la fin des années quatre-vingt, les évaluations de CE2 et de sixième). Les évaluations nationales et locales sont de plus en plus nombreuses, ces dernières étant souvent produites à la demande des cadres intermédiaires de l'Éducation nationale. Elles sont tour à tour, ou simultanément, des outils permettant de mesurer l'efficacité de l'enseignement dispensé, tout particulièrement en ZEP, des outils de suivi des apprentissages (certains cahiers de grande section de maternelle indiquent chaque mois aux parents ce qui a été non acquis, partiellement acquis ou acquis par leurs enfants) ou encore des outils diagnostiques ou de dépistage des troubles de l'apprentissage. Ces différents types d'évaluation finissent, quels que soient leurs objectifs, par se contaminer les uns les autres et cela d'autant plus que certaines épreuves se retrouvent à peine modifiées d'un matériel à l'autre. Beaucoup d'enseignants et de membres des réseaux d'aides spécialisées aux élèves en difficulté (RASED) finissent par ne plus y voir qu'une demande bureaucratique pesante qui ne leur semble d'aucun secours dans leur travail quotidien.

Le brouillage qui règne du côté des enseignements à dispenser se retrouve du côté de la pédagogie : quand les plus hautes autorités ministérielles formulent l'injonction de se recentrer sur l'essentiel, comment ne pas comprendre que, pour y parvenir, le mieux est de revenir aussi aux méthodes pédagogiques d'antan grâce auxquelles les maîtres du passé sont censés être arrivés à transmettre les savoirs de base à tous leurs élèves ? Mais tout en transmettant lesdits savoirs, les enseignants modernes sont sommés de "placer l'élève au centre des apprentissages ${ }^{7}$ ». Ils doivent produire des pratiques innovantes tout en puisant leur inspira- tion dans la tradition pédagogique qui aurait sa source dans l'école républicaine du début $d u x x$ siècle. La nouvelle version de la querelle des Anciens et des Modernes qui sévit dans les médias à propos de l'école et de ses missions pèse sur tous les enseignants, mais elle le fait tout particulièrement sur ceux des ZEP : ces derniers doivent tout à la fois inventer une nouvelle école capable de répondre aux besoins des élèves contemporains des quartiers populaires et reproduire dans ce qu'elle avait de meilleur l'école de Jules Ferry, fantasmée comme l'école du peuple.

Qu'on ne s'étonne donc pas de voir tous les modèles pédagogiques coexister dans la même école, d'un maître à l'autre, voire, pour un même maître, d'une discipline à l'autre : un même enseignant de CP peut par exemple utiliser une méthode de lecture ancienne ${ }^{8}$ qui ne sollicite chez les élèves que la mémorisation de relations grapho-phoniques et, en sciences, construire une activité innovante, comme celles recommandées par les promoteurs du dispositif "La main à la pâte 9 ", qui elles-mêmes reproduisaient (l'ignorant parfois) des démarches déjà introduites dans les classes par des mouvements pédagogiques avant et après la Seconde Guerre mondiale. La difficulté scolaire qu'affrontent les maîtres de ZEP, par l'insécurité qu'elle crée chez eux, accentue ces phénomènes, au détriment d'élèves qui plus que d'autres ont besoin de régularité et de clarté dans l'organisation et la conduite du travail scolaire.

\section{Écarts entre les curriculums prescrits et les curriculums réalisés en ZEP et hors ZEP}

À ces contradictions qui pèsent sur tous les enseignants, mais plus particulièrement sur ceux qui exercent en ZEP, s'ajoutent les très fortes attentes des parents à l'égard de l'école, attentes qui ne se manifestent pas de la même manière selon les classes sociales. On pourrait penser que l'addition de toutes ces contradictions, attentes et exigences ne produise pas le même effet en ZEP et en dehors, que les enseignants n'opèrent pas les mêmes choix dans leur travail quotidien selon qu'ils exercent en ZEP ou non et que se crée progressivement une différenciation marquée dans les pratiques des classes entre les ZEP et le reste du système éducatif. Or la réalité semble beaucoup plus nuancée, surtout en primaire. II n'existe malheureusement que fort peu d'études quantitatives menées à grande échelle qui permettraient d'avoir une idée précise de cette réalité ${ }^{10}$. L'auteure de ces lignes est donc obligée pour ce faire de s'appuyer sur son expérience des classes ordinaires et sur le fait qu'elle a été associée à la mise en place des ZEP et à leur suivi ainsi 
qu'au travail des CEFISEM (Centres de formation et d'information pour la scolarisation des enfants de migrants), tant sur le plan local qu'au niveau national, et qu'elle a participé à de très nombreuses sessions de formation destinées aux enseignants ayant en charge des élèves en grande difficulté, où elle les a entendus exprimer leurs interrogations. La sélection des notions à enseigner, l'utilisation du matériel pédagogique et le mode d'organisation de la classe dépendent-ils de critères différents selon qu'on est en ZEP ou non?

\section{Des exigences d'apprentissage de plus en plus précoces}

L'investissement toujours plus grand des parents dans l'école et l'évolution des représentations collectives sur les capacités des jeunes enfants a produit un lent mais continu glissement de l'aval vers l'amont des contenus d'apprentissage, notamment de l'école élémentaire vers l'école maternelle, glissement permis et accentué par la structuration du système en cycles, dont le premier est à cheval sur la dernière classe de l'école maternelle (grande section) et la première de l'école élémentaire (CP). Les disciplines comme le français dont les progressions ne sont pas stabilisées (Nonnon, 2010) et dont les savoirs à enseigner ne font pas l'objet d'un consensus sont particulièrement touchées : on enseigne fréquemment à des élèves de grande section comme on le ferait à des élèves de cours moyen, à reconnaître l'auteur d'un livre et à ne pas le confondre avec le narrateur de l'histoire ni avec l'éditeur ou le dessinateur. On peut leur apprendre à retrouver une quatrième de couverture, voire à distinguer un point de vue narratif d'un autre... On les confronte au principe des mots croisés ou à l'utilisation de calendriers divers ou de tableaux et d'affiches dont l'agencement est complexe (Bautier, 2005 ; Joigneaux, 2009a, 2009b).

En début de CE2, des élèves sont censés être capables de repérer dans un texte, en s'appuyant sur les marques verbales, les différents plans temporels de la narration, ou, en s'aidant des marques graphiques du pluriel et du féminin, d'inférer des informations sur le nombre et le genre des personnages. L'exemple le plus manifeste de précocité réside dans le fait que l'apprentissage du code alphabétique occupe désormais la grande section de maternelle (au lieu du cours préparatoire). Mais de tels phénomènes de glissement vers l'amont de contenus, d'exigences et de tâches à la fois de plus en plus précoces et peu hiérarchisés, peu régulés, s'observent à tous les niveaux de la scolarité élémentaire et concernent bien d'autres domaines ou disciplines que l'enseignement de la lecture (fran- çais, histoire, sciences de la vie par exemple). Derrière les curriculums prescrits se dissimule une grande confusion des curriculums réels dont ni l'institution, ni les parents, ni les enseignants, ni bien sûr les élèves n'arrivent à se faire une idée claire. On pourrait s'attendre à ce que les ZEP soient moins touchées par un tel phénomène, parce que la pression des parents $y$ serait moins forte et parce que les difficultés supposées de leurs élèves inciteraient les enseignants à se centrer davantage qu'ailleurs sur ce qu'ils estiment plus facile ou plus utile à leurs élèves. Or en la matière, au moins au début de la scolarité, il n'en est rien, sans doute parce que le souci de proposer des activités intéressantes et variées se superpose à celui d'aller à l'essentiel. Et l'on constate que les grandes sections de maternelle se ressemblent, en ZEP et hors ZEP.

\section{Choix et usages des outils pédagogiques en ZEP et hors ZEP}

Les produits de l'édition scolaire et les ressources disponibles sur Internet où les enseignants mutualisent leurs préparations d'activités et de séquences sont surabondants et d'une extrême diversité. Ils semblent être utilisés indifféremment en ZEP et hors ZEP. Ainsi les enseignants de ZEP ne paraissent-ils avoir aucun critère particulier pour sélectionner un manuel ou une fiche de travail individuel plutôt qu'un autre. L'exemple des manuels de lecture est particulièrement éclairant : sur les trente dernières années, successivement trois ou quatre manuels se sont retrouvés en position dominante durant une période relativement courte et ont été remplacés par une poignée d'autres manuels à leur tour dominants. Ils sont aussi présents en ZEP qu'en dehors. Ce qui l'emporte alors dans le débat permanent sur les méthodes de lecture (le " travail sur le sens " ou le "travail sur le code ") prime dans l'esprit des enseignants de ZEP sur tout autre critère apte à déterminer le choix des méthodes les plus adaptées à leurs élèves (comme par exemple la rapidité des progressions ou l'appréhension par leurs élèves de l'univers de référence des textes). Or, les enseignants de CP en ZEP soulignent très souvent que leurs élèves usent d'un lexique très peu varié, ce qui serait une des raisons pour lesquelles nombre d'entre eux rencontrent des problèmes dans l'apprentissage de la lecture. Les maîtres devraient alors retenir celle des méthodes qui présente la plus faible dispersion lexicale (des méthodes très semblables par ailleurs sont extrêmement différentes de ce point de vue). Mais au contraire, tout à leur souci d'enrichir le vocabulaire de leurs élèves, souci qui se manifeste dans toutes les activités proposées (du travail sur le code à la lecture d'albums de jeunesse), ils choisissent souvent des matériels uti- 
lisant un lexique abondant, varié et dont la référence est souvent inconnue de leurs élèves. Le recours à des fichiers accentue encore ce phénomène. S'il est difficile de s'affranchir de la progression imposée par un manuel, ce n'est pas le cas avec ces derniers. Une fiche présentée dans un fichier comme devant être utilisée au troisième trimestre de grande section peut être sélectionnée et utilisée par un enseignant, pour des raisons thématiques, au deuxième trimestre : la " semaine du goût » peut conduire l'enseignant à sélectionner une fiche où sont représentés différents aliments sucrés ou salés sans tenir compte de la place qu'occupait la fiche dans le fichier auquel elle a été empruntée.

La diversité des matériaux qu'utilisent les enseignants de grande section et de CP les met souvent dans l'impossibilité de construire un enseignement raisonné du lexique. Le vocabulaire est introduit dans la classe au hasard des besoins que créent en la matière les activités successives. Les logiques thématiques, phonographiques, morphosyntaxiques s'entrecroisent de manière inextricable. L'absence de tout enseignement méthodique du lexique est loin d'être propre aux ZEP, mais il est particulièrement préjudiciable aux élèves qui ont le plus de besoins en la matière. D'autres exemples d'agencements de ce type, qui ne peuvent qu'être préjudiciables aux élèves les plus fragiles, pourraient être développés concernant d'autres disciplines.

Cet éclectisme didactique permet à l'enseignant de concilier des impératifs qui, sans lui, pourraient passer pour contradictoires, tels que, par exemple, se focaliser sur les apprentissages supposés être les plus simples, souvent désignés par le terme «b.a.-ba », ou diversifier les situations pour susciter l'intérêt des élèves et les initier à des pratiques sociales et culturelles nouvelles - par exemple, travailler certaines fonctions de l'écrit en construisant une séance autour des procédures du vote employées dans des élections locales ou nationales (Laparra \& Margolinas, 2010).

\section{Organisation du travail en classe et types d'activités}

À la fois aidés par les possibilités matérielles de produire ou reproduire aisément tout type de document fabriqué ou non pour un usage scolaire, influencés par les modèles pédagogiques faisant appel à la curiosité des élèves et à leur capacité d'implication dans les tâches proposées, les enseignants à tous les niveaux de la scolarité diversifient les modes d'organisation du travail dans la classe. II est dès lors fréquent de voir, dans une même classe, se succéder une phase de tra- vail avec la classe entière, une phase de travail en petits groupes de taille variable et une phase de travail individuel. Chaque maître articule ces phases d'une manière qui lui est propre (ordre, durée...) et utilise tel type d'organisation plutôt qu'un autre selon qu'il s'agit d'une activité dite « de découverte », d'application, de réinvestissement, d'évaluation, un même type d'activité ne s'accompagnant d'ailleurs pas forcément toujours du même mode d'organisation. La grande section privilégie l'organisation en ateliers qui peuvent correspondre aussi bien à un travail de groupe qu'à un travail individuel sur fiches, et qui conduisent fréquemment à un accroissement non seulement des exigences que l'on a à l'égard des élèves, mais aussi de leur caractère implicite et opaque pour les élèves les moins familiarisés avec l'univers scolaire (Joigneaux, 2009a, 2009b). Les différences qui existent d'une classe à une autre s'observent aussi bien en ZEP qu'en dehors, en primaire ou au collège. Le recours au travail en groupe ne sert pas souvent à faire face aux problèmes posés à l'enseignant par l'hétérogénéité de ses élèves, et cela tout aussi bien en ZEP et en dehors ; les groupes dits de niveau y sont également peu fréquents, hormis dans les enseignements de langue vivante.

De la même manière alternent des phases d'enseignement frontal et des phases où les élèves, travaillant seuls, en groupe, voire en classe entière, doivent mobiliser les ressources langagières, cognitives et culturelles qui sont supposées être les leurs et confronter les procédures employées pour mener à bien une tâche (comme comparer et justifier les différents classements de données qu'ils ont effectués). Le repérage et l'identification de ces activités, de leurs enchaînements, de leurs rapports sont d'autant plus malaisés que les noms par lesquels elles sont désignées varient, d'une discipline à l'autre, voire d'une activité ou d'un moment à l'autre. La phase d'institutionnalisation des savoirs, quant à elle, peut être très peu développée, voire inexistante, elle peut ne comporter aucune trace écrite et quand elle en comporte une, celle-ci peut être élaborée par le maître seul, écrite au tableau et recopiée par les élèves ou dictée ; elle peut aussi l'avoir été par les élèves eux-mêmes, collectivement ou individuellement, le maître sélectionnant alors la proposition qui lui semble la meilleure. On retrouve de telles variations à tous les niveaux de la scolarité et d'une discipline à une autre, en ZEP aussi bien qu'en dehors. Selon le type de savoir enseigné, un même enseignant peut susciter chez ses élèves des réflexions métalinguistiques ou métacognitives, qu'il soit ou non en ZEP. Les enseignants du primaire disent le faire plus souvent dans les disciplines où ils ont des savoirs académiques assurés du fait de leur formation universitaire initiale et 
s'interdire de procéder ainsi dans les disciplines qu'ils pensent moins bien maîtriser.

\section{L'école, machine à scolariser le monde extérieur}

L'école contemporaine doit à la fois assurer une sanctuarisation de son espace et s'ouvrir sur son environnement ; elle doit être une forteresse, mais largement tournée vers la cité et le monde. Tout peut être support d'apprentissage au terme d'un processus de scolarisation : la littérature de jeunesse est devenue à la fois un objet et un moyen d'enseignement, subissant à son tour le même traitement que jadis la littérature patrimoniale ou plus récemment les écrits sociaux non littéraires. Mais les maîtres continuent bien sûr à utiliser avec leurs élèves des supports qui n'ont d'existence que scolaire et qui sont fabriqués uniquement à des fins d'enseignement. Dans une même séance, les élèves peuvent dès lors travailler sur un mode d'emploi ou une recette et manipuler plusieurs phrases n'ayant aucun lien entre elles. Un univers inventé aux seules fins scolaires et un univers importé du monde se côtoient à l'école, d'une situation à l'autre, parfois même dans la même situation, sans que soient travaillés et élucidés les spécificités, les règles et les normes propres à chacun de ces univers, leurs différences et leurs possibles rapports. Une telle coexistence, implicite et peu régulée, est présente en ZEP et hors ZEP, mais elle est sans doute plus dommageable pour des élèves qui n'ont pas tous les outils pour comprendre quelles sont les règles régissant ces deux types d'univers et les passages de l'un à l'autre. Quand les élèves présentent des signes de rupture avec l'école, les maîtres de ZEP peuvent en outre être tentés de rapprocher au maximum l'école de son environnement au point de reléguer parfois au second plan les exigences scolaires, au profit du souci de rendre les situations et activités plus attractives ou moins artificielles (Kherroubi \& Rochex, 2004).

\section{UN TRAITEMENT PARTICULIER DES ÉLÈVES DE ZEP}

En ZEP comme hors ZEP, on est donc très loin du fonctionnement largement imaginaire de l'école de Jules Ferry qui aurait fait que dans toutes les écoles, les élèves effectueraient la même tâche à la même heure. Une extrême diversité dans les activités est la marque du système : d'une classe à l'autre ou à l'intérieur d'une même classe coexistent des activités obéissant à des logiques différentes ; une même activité peut être soumise elle-même à plusieurs logiques.
Une telle hétérogénéité est sans doute pour une part inévitable. Mais, faute de régulation et de mise en cohérence du point de vue du travail et des apprentissages attendus des élèves, elle est source d'insécurité pour les différents acteurs de l'école et source d'instabilité pour tous les élèves, insécurité et instabilité qui sont particulièrement dommageables quand elles s'ajoutent à celles qui sont présentes dans l'environnement socio-économique des élèves issus des familles et des quartiers les plus précarisés. Identiques en ZEP et hors ZEP, ces phénomènes n'y produisent pour autant pas les mêmes effets... Mais les ZEP présentent en outre des phénomènes qui, même s'ils ne sont pas toujours spécifiques, y sont particulièrement marqués et dont les effets s'ajoutent aux précédents.

\section{La «pédagogie de la réussite »}

Si d'une manière générale les parents et l'institution attendent que tous les élèves soient mis en situation de réussite à l'école, dans le reste du système éducatif cette obligation ne domine pas, voire ne gouverne pas exclusivement la conduite des tâches et ne devient pas une fin en soi à la différence de ce qui arrive très souvent en ZEP. Les enseignants y font en sorte de ne pas ajouter aux difficultés supposées de leurs élèves et ils mettent tout en œuvre pour que ceux-ci mènent à bien la tâche qui leur est proposée. S'installe alors trop souvent la confusion entre l'effectuation réussie de la tâche et la construction effective des savoirs : le découpage de l'activité en micro-tâches à réaliser les unes après les autres, le rythme de travail proposé et les aides fournies par les enseignants permettent fréquemment à l'élève de faire ce qui est attendu de lui sans qu'il n'y ait aucun apprentissage réel, ce dont témoignent les écarts importants qui apparaissent entre les performances des élèves quand ils travaillent sur une fiche individuelle en atelier ou en grand groupe, situations où ils peuvent bénéficier de nombreuses interactions avec le maître et leurs camarades, et les performances qui sont les leurs lors de la passation d'épreuves d'évaluation où ils sont livrés à eux-mêmes.

Quand apparaît un décalage trop important entre la vitesse à laquelle travaille une part de leurs élèves et celle qui leur semblerait normale à leur âge, les enseignants agissent rarement sur ce qui rend leurs élèves aussi lents (qui tient par exemple à une écriture mal automatisée et une mauvaise maîtrise de l'organisation de l'activité). Faute de pouvoir hiérarchiser ce qui est central et ce qui est secondaire, de pouvoir alléger ou prendre en charge le secondaire pour rendre les élèves plus disponibles à l'essentiel, ils ne modifient guère les 
contraintes et l'organisation de l'activité, mais préfèrent ou ne peuvent faire autrement que de régler le rythme du travail dans la classe sur celui de leurs élèves en étirant le temps et en faisant travailler leurs élèves de plus en plus lentement ${ }^{11}$, ce qui les contraint bien souvent, devant l'écoulement du temps, à devoir renoncer à certains éléments pourtant initialement prévus (" Ça, ne le fais pas maintenant », "Passe directement au point suivant ", entend-on souvent dans ces classes). Ne vaut-il pas mieux, disent-ils alors, que tous les élèves viennent à bout de la tâche qui leur est proposée, quitte à ce que celle-ci soit d'autant plus restreinte que l'élève est plus en difficulté ? Toujours portés par ce même souci de réussite, ils guident très fortement leurs élèves, leur apportant une aide le plus souvent individuelle lors de l'effectuation de leur travail. II n'est pas rare d'observer, à tous les niveaux du système éducatif, des classes où les élèves sont habitués à demander une aide à chaque étape de l'activité proposée et à solliciter la validation de ce qu'ils font, non pas une fois la tâche finie mais au fur et à mesure de son déroulement.

Cette dépendance à une aide permanente, souvent décrite en termes de manque d'autonomie, n'est pas particulière aux élèves de ZEP, mais elle est massive chez eux et elle peut avoir deux conséquences opposées en ce qui concerne les devoirs faits à la maison : soit les enseignants continuent d'en donner et les élèves n'arrivent pas à effectuer le travail personnel attendu d'eux, faute de disposer chez eux de cette aide, et ils peuvent alors être accusés de ne pas vouloir faire le travail demandé ; soit les enseignants, conscients de cette difficulté, renoncent complètement aux devoirs à la maison. Dans les deux cas, les élèves ne voient pas le travail fait à l'école être repris et renforcé par un travail extrascolaire, à la différence des élèves qui trouvent au sein de leur famille l'aide dont ils ont besoin. Les dispositifs d'aide aux devoirs, nombreux et variés, essayent de résorber cette inégalité mais n'y arrivent que très imparfaitement, faute d'être suffisamment intensifs.

Si on peut avoir l'impression, en observant le fonctionnement des classes de ZEP, que les contenus d'enseignement n'y sont pas les mêmes que dans le reste du système éducatif, ce n'est pas parce que les enseignants s'affranchiraient du respect des programmes mais parce qu'ils s'efforcent de les exécuter au plus près de ce qu'ils croient être les capacités de leurs élèves. Tout au long de la scolarité, ils essayent de ne pas céder sur ce qu'ils pensent être l'essentiel : les classes de CP en ZEP où les enseignants ne s'acharnent pas à construire la maîtrise du code alphabétique ou de la numération sont des exceptions rarissimes. Les maîtres ne renoncent à traiter certaines parties des programmes que quand ils ne trouvent pas de solution pour le faire. L'enseignement de la littérature au collège en fournit un bon exemple : les comédies de Molière ne peuvent être présentées dans des versions adaptées. L'obstacle de la langue est tel que certains enseignants ne les proposent pas à leurs élèves ; mais ces mêmes enseignants leur feront lire des romans courtois, parce qu'ils peuvent alors utiliser des traductions de ces œuvres en français moderne.

\section{Une « dé-didactisation " de la difficulté scolaire}

Placés dans une situation d'anomie généralisée où ils doivent effectuer seuls les choix qui leur permettent de conduire leur action, les enseignants de ZEP, plus encore que leurs collègues des établissements situés hors ZEP, parce que les difficultés résistent à tous leurs efforts, éprouvent souvent un sentiment d'impuissance professionnelle qui leur fait reporter la responsabilité de l'échec sur leurs élèves. On assiste alors à une "dé-didactisation » des problèmes qui sont source de difficultés et d'inégalités d'apprentissage. Une telle " dé-didactisation " a caractérisé nombre de discours sur les ZEP depuis leur création.

À une phase qui a pris fin dans les années quatrevingt-dix, où étaient mis en avant des facteurs collectifs de type sociologique pour rendre compte de l'échec scolaire, a succédé une phase où sont massivement invoqués des facteurs individuels, de type psychologique ou médical. Ils n'ont pas remplacé les premiers mais se sont ajoutés à eux ${ }^{12}$. Pour expliquer les difficultés que manifeste un élève, on peut dire de lui tout à la fois que "sa famille ne s'intéresse pas à l'école ", qu' " on ne surveille pas ses devoirs ", mais simultanément qu'il est "lent, timide, pas autonome, incapable d'une attention soutenue, n'obéissant jamais ", qu'il est «immature ", " inhibé ", "surprotégé ", qu'« il manque de repères " ou encore qu'il est " dyslexique ", " dysorthographique ", " dyscalculique »... Selon les interlocuteurs, le diagnostic d'échec varie, mais les comportements des élèves qui leur valent ce type de jugement ne sont pratiquement jamais mis en relation avec des besoins d'apprentissage. On entend rarement un enseignant dire : "Certes, il est lent, mais il l'est surtout quand il doit écrire quelque chose "; "S'il écrit lentement, c'est parce qu'il tient mal son stylo et qu'il n'a pas automatisé l'engendrement de certaines lettres "; ou encore : " II est agité mais son agitation se manifeste à des moments particuliers, avant d'entrer dans la tâche ou après en avoir effectué le premier élément. II est dépendant d'une 
aide permanente. II faut que je lui donne certains outils qui lui font défaut, par exemple sur le plan de l'organisation de sa fiche, pour qu'il apprenne à travailler seul », etc.

\section{NÉCESSITÉ DE DIDACTISER LA DIFFICULTÉ SCOLAIRE}

L'existence d'un échec scolaire important dans les ZEP et sa persistance (quelles que soient les politiques mises en œuvre) conduisent à se demander s'il ne faut pas procéder au contraire à une forte didactisation des problèmes rencontrés par les élèves dans leur scolarité et mettre en question les processus dominants d'adaptation des enseignements à ce qu'on pense être les particularités des élèves. Pour ce faire, il est sans doute nécessaire de s'interroger sur l'analyse qui est faite des différences indéniables qui s'observent entre les élèves, notamment au début de la scolarité. Alors que leurs compétences linguistiques et leurs connaissances du monde sont variables de l'un à l'autre, cela rend-il impossible la réalisation d'apprentissages identiques pour tous ? Quatre phénomènes jouant simultanément le laissent croire et font qu'en maternelle et au CP on ne voit pas que pratiquement tous les élèves ont les capacités requises pour réussir les apprentissages prescrits. Les exemples qui suivent relèvent tous de la didactique du français, mais d'autres pourraient être fournis dans de nombreuses disciplines.

\section{Sous-estimation des capacités des élèves des milieux populaires}

Faute de disposer d'études suffisamment nombreuses sur ce que disent effectivement ces élèves lors des activités ordinaires, on affirme souvent à tort que leur usage de la langue dans la communication quotidienne ne leur permet pas de réaliser les verbalisations qu'exigent les opérations cognitives en jeu dans les apprentissages. Or, ils peuvent procéder à des comparaisons, à des classements, des appariements, etc., et rendre compte de ce qu'ils font. N'importe quel élève peut dire que deux objets "ne sont pas pareils " aussi bien qu'ils « sont différents "; tous sont capables de passer d'un énoncé comme : "Ces deux objets n'ont pas la même couleur, la même forme... " à un énoncé tel que : "La couleur de ces deux objets est différente ", pour peu qu'on leur apprenne à le faire et qu'on l'exige d'eux ; rien dans leur compétence linguistique ne leur interdit de le faire. De la même manière, contrairement à une idée reçue, il n'est pas nécessaire de disposer d'un lexique abondant ${ }^{13}$ pour apprendre à lire, il suffit que les supports utilisés pour ce faire n'utilisent qu'un lexique commun à tous les élèves ; et ce lexique commun est souvent plus varié qu'on ne le croit et largement suffisant pour travailler sur des énoncés intelligents et motivants. Les activités permettant d'arriver à lire efficacement exigent des élèves un travail suffisamment prenant pour qu'on évite d'y mêler d'autres objectifs comme l'enrichissement du vocabulaire des élèves, lesquels peuvent être tout à fait légitimes par ailleurs.

Quand on s'interroge sur le rapport à l'écrit qu'ont les familles de milieu populaire, on ne cherche guère qu'à savoir si elles en ont justement des usages " experts " (lire des magazines, écrire des lettres), ce qui n'est pas souvent le cas, pas plus que ce n'est le cas de certaines familles favorisées sur le plan économique. On ne s'aperçoit pas qu'il est fréquent qu'elles aient certains usages de la littératie non linguistique : elles vivent en effet comme toutes les autres dans un univers organisé à l'aide des ressources non linguistiques de l'écrit (lignes, colonnes...). De la piscine au supermarché, les corps se meuvent dans un espace de littératie et les objets du monde y trouvent leur place (Privat, 2010). Tous les élèves sont capables d'ordonner une collection d'objets en se servant de ces ressources pour peu qu'on les ait rendus sensibles au fait qu'il y a un intérêt à le faire : il est ainsi plus facile de procéder à un dénombrement des objets d'une collection, sans courir le risque de se tromper, si on a pris la peine de les ranger préalablement en lignes et en colonnes. Mais il n'est pas rare de voir au cours moyen des élèves qui ne disposent pas de ces procédures - ce qui les gêne beaucoup dans les tâches habituelles - alors qu'ils auraient parfaitement été capables de les acquérir dès la maternelle, pour peu qu'on les leur ait enseignées. Les élèves ne peuvent réussir les tâches proposées en maternelle et au CP que s'ils disposent de ces savoirs de la littératie, que rien au plan socioculturel ne leur interdit d'acquérir et qu'ils sont parfaitement capables de mettre en jeu de manière pertinente. II s'agit souvent de savoirs qui relèvent à la fois de la didactique des mathématiques (sur le plan de l'énumération) et de la didactique du français, sans pour autant être toujours thématisés et travaillés par ces didactiques. Dès lors, quand les élèves les rencontrent - ce qui n'est pas fréquent et advient le plus souvent dans des séances dites de méthodologie -, ils ne leur sont alors pas présentés comme des savoirs disciplinaires et restent souvent implicites. 


\section{Dévalorisation de l'univers de l'oralité}

Au lieu de valoriser les connaissances de l'oralité qui sont les leurs, il arrive même qu'on les dévalorise. Donnons-en quelques exemples. Tous les jeunes élèves, quel que soit leur milieu socioculturel, manifestent un même intérêt et une même aptitude pour toutes les activités verbales routinisées qui s'accompagnent d'une mise en scène corporelle. Ils prennent un plaisir avéré à oraliser les comptines que sont pour eux les suites de nombres, à compter de deux en deux, à chanter certaines comptines alphabétiques. Ils déroulent ces suites verbales en s'aidant de leurs doigts, en en soulignant la mélodie par des mimiques ou en marquant les cadences par de légers coups frappés sur leurs genoux, sur leur front ou dans la paume d'une main. L'école, quand elle recourt à de telles pratiques, les présente souvent comme une récompense pour un travail bien fait, prenant place dans un moment de détente. Elle n'explicite pas ce que les élèves pourraient acquérir comme savoirs grâce à elles. Pour les élèves comme pour le maître, elles sont un jeu, pas un travail, ce qui explique qu'elles se raréfient dès que l'école se vit uniquement comme un lieu d'apprentissage. Ainsi, non seulement l'école ne donne pas un statut scolaire à tout ce qui appartient à l'univers de l'oralité, mais elle ne renforce pas celles des connaissances de l'oralité dont l'élève aura besoin quand il passera dans l'univers de la littératie. Comment produire à l'écrit la liste des jours de la semaine si on ne sait pas produire à l'oral la suite de ces jours ; comment se servir de l'ordre alphabétique pour organiser une collection si on n'a pas en mémoire, parfaitement stabilisée, la suite orale des lettres de l'alphabet?

Mais plus grave encore, l'école, toute à son souci de prouver la supériorité de l'écrit sur l'oral, apprend aux élèves à se défier de leurs connaissances orales, alors même que celles-ci sont assurées (Bachmann, 1977). Tous les élèves, même ceux qui sont les plus en difficulté, mémorisent aisément les résultats d'une opération de dénombrement qu'ils viennent d'effectuer, quand il s'agit de deux ou trois nombres inférieurs à dix (par exemple : "Il y a trois étiquettes rouges, deux vertes et six bleues "). II arrive qu'on entreprenne alors, contre l'évidence, de leur montrer qu'ils ne peuvent s'en souvenir que s'ils les écrivent ; il suffit pour ce faire de demander aux élèves réputés les plus faibles de la classe s'ils sont sûrs de ce qu'ils disent, ces derniers finissant par admettre que non. Se trouve alors progressivement dévalorisé l'univers de l'oralité qui est pourtant celui dans lequel les élèves et leur famille se meuvent, puisqu'il leur est présenté comme imparfait et instable. Au travers de leurs connaissances orales, ce sont tous leurs usages langagiers et leur rapport à la langue qui sont petit à petit disqualifiés, ce qui les prive eux ainsi que leurs enseignants de ressources sur lesquelles ils pourraient prendre appui pour opérer un passage dans l'univers de la littératie.

\section{Types d'acquis préscolaires des élèves de milieu aisé}

Faute d'avoir observé comment les élèves des classes favorisées ont construit certaines des aptitudes qui sont décisives dans les apprentissages supposant une activité réflexive, on ne remarque que leur capacité à adopter un point de vue formel sur la langue, capacité qui semble faire souvent défaut aux autres. On en déduit que leur famille aurait construit chez eux ce rapport à la langue, parce qu'elle aurait, à la différence des familles de milieu populaire, des usages de la langue autres qu'éthico-pratiques (Lahire, 1993).

Or, si ces familles donnent bien une avance dans les apprentissages graphiques à leurs enfants, elles le font en leur montrant à reproduire à l'identique, comme pour un jeu de construction, un ensemble qui est composé d'éléments différents ; l'assemblage qu'ils doivent reproduire à l'aide d'un modèle n'est pas fait de pièces matérielles, il est fait de lettres et le résultat obtenu est un mot. Les enfants apprennent ainsi à reconnaître un nombre restreint de mots écrits, qui ne sont pas forcément les mêmes d'une famille à une autre (" papa ", " maman », les prénoms des enfants de la famille ou des parents, un ou deux noms d'animaux, des mots comme " maison " ou « soleil »...) et ils les reproduisent avec ou sans modèle, en apprenant progressivement à épeler les lettres qui les composent. II s'agit là d'activités fortement routinisées, effectuées dans un climat affectif sécurisant ; elles appartiennent pour les acteurs adultes et enfants au monde matériel.

II n'est pas rare de voir des élèves procéder ainsi entre eux durant la phase d'accueil en maternelle : ils écrivent sur leur ardoise le prénom des élèves qui sont dans le même groupe qu'eux en prenant comme modèles les étiquettes collées sur leur table, ils les encadrent puis effacent ce qu'ils viennent de faire et recommencent l'opération à l'identique à plusieurs reprises. La répétition jour après jour de ces activités de recopiage les dote d'une mémoire lexicale extrêmement restreinte mais assurée ; elle porte sur des termes ayant un poids affectif et une importance référentielle ; elle leur permet de commencer à repérer des associations régulières de lettres, ouvrant ainsi la porte à l'ana- 
lyse du code. La moyenne et la grande sections de maternelle pourraient procéder ainsi avec les élèves de milieu populaire, qui ne disposent pas, ou moins, d'une telle mémoire lexicale. Mais elles ne le font que rarement, ce qui a pour conséquence que ces élèves se trouvent démunis, à la différence de leurs camarades plus favorisés, quand commence le travail d'analyse de la chaîne sonore et graphique : ils ne peuvent s'appuyer sur une mémoire lexicale que des recopiages beaucoup trop dispersés n'ont pas pu construire, alors que l'on attend d'eux qu'ils soient d'emblée capables de trouver ce qui est semblable et différent dans la chaîne sonore. Si leurs camarades de milieu favorisé y parviennent mieux qu'eux, c'est qu'ils peuvent s'appuyer sur ce qu'ils ont appris à comparer et à rapprocher, en écrivant sans cesse une douzaine de mots.

\section{Relation d'aide fortement différenciée}

Les supposées différences d'aptitude entre les élèves expliquent que les enseignants ne fournissent pas la même aide à tous leurs élèves et qu'ainsi ils ne les exposent pas aux mêmes apprentissages, ou qu'ils ne le fassent pas de la même manière, créant ainsi de la différenciation scolaire, source d'inégalités. Fournissons un exemple très simple de ce phénomène qui est constant dans les classes, à tous les niveaux et dans toutes les disciplines. Lors d'un travail de groupe au $\mathrm{CP}$, les élèves doivent produire à l'écrit la liste des ingrédients d'une recette. Aucun élève ne sait écrire le mot "sucre ". Aux bons élèves qui lui demandent son aide, la maîtresse fournit directement la réponse en leur écrivant elle-même le mot, leur permettant ainsi de se concentrer sur ce qui est essentiel dans l'activité, dresser une liste et comprendre l'intérêt qu'il y a à le faire. Aux élèves qui sont considérés comme de mauvais lecteurs, elle leur dit d'essayer de trouver comment le mot s'écrit, en se le répétant à voix basse. Si elle procède ainsi, c'est sans doute parce qu'elle pense qu'il faut saisir toutes les occasions qui se présentent pour leur faire travailler les correspondances phono-graphiques, dont la maîtrise insuffisante est censée être à la source de toutes leurs difficultés. Les élèves en question n'arrivent bien évidemment pas à transcrire "sucre " : en échec dans une tâche apparemment très simple, ils n'acquièrent aucune connaissance sur la liste.
Les élèves les plus faibles sont ainsi souvent cantonnés dans des activités dites de bas niveau, parcellisées et répétitives : sans fin ils copient des modèles, procèdent inlassablement à des dénombrements ; ils s'appliquent à effectuer toutes les tâches matérielles avec la plus grande minutie et finissent par perdre le sens des apprentissages. Ils peuvent d'ailleurs n'être que rarement exposés aux apprentissages. Le processus d'adaptation des situations et des contenus aux capacités supposées des élèves peut ainsi conduire à ce qu'on ne repère pas quelles sont les connaissances que les élèves mettent en jeu pour réaliser ce qu'on attend d'eux, ni quelles sont les connaissances auxquelles ils seraient capables de recourir si on les sollicitait à le faire, ni même quelles sont les connaissances nouvelles qui sont nécessaires à la construction des apprentissages visés. Ce phénomène, qui a été décrit ici essentiellement au début de la scolarité et en français, affecte toutes les disciplines et se reproduit à tous les niveaux du système éducatif, avec bien sûr des différences qui tiennent aux effets de cumul des difficultés (Rochex \& Crinon, 2011).

\section{CONCLUSION}

L'observation de ce qui se passe en ZEP permet d'affirmer que les difficultés scolaires des élèves ne leur sont pas imputables, ni à eux ni à leurs familles. C'est bien la manière dont sont construites les situations d'apprentissage et conduites les activités qui est à la source de leurs problèmes. Cela ne revient pas à mettre en cause la compétence professionnelle des enseignants. Ceux-ci ne pourront procéder autrement qu'ils le font, surtout en ZEP, tant que l'ensemble des acteurs de l'école n'aura pas la possibilité d'apprendre à s'appuyer sur les résultats de minutieuses études de terrain produites à leur demande pour résoudre le conflit existant entre la nécessité républicaine de proposer à tous les élèves le même enseignement et l'exigence démocratique de prendre en compte leur diversité.

Marceline Laparra laparra@univ-metz.fr Université de Lorraine, CREM 
1 Concernant par exemple l'enseignement du français, voir Pratiques (1999).

2 Même si peut y ressembler l'injonction faite récemment aux ZEP de conduire les meilleurs de leurs élèves dans les filières d'excellence.

3 Ces injonctions et recommandations sont récurrentes, depuis les rapports du premier " groupe de pilotage " de la politique ZEP et les premières universités d'été organisées avec le soutien du ministère dans les années quatre-vingt jusqu'aux rapports des inspections générales (Bachmann, Duro-Courdesses, Chauveau et al., 1983 ; Moisan \& Simon, 1997 ; Armand \& Gille, 2006).

4 Voir les travaux de Jean-François Halté dans le cadre de l'INRP ou de Pratiques pour le français, ou encore les travaux publiés par certains IREM (Institut de recherche sur l'enseignement des mathématiques) pour les mathématiques.

5 On a pu distinguer " trois âges " dans cette histoire (voir Rochex, 2010 ; Frandji \& Rochex, 2011).

6 C'est Gérard Chauveau qui a introduit le premier cette acception de la notion d'" excellence " à propos des ZEP (cf. notamment Chauveau, 2000).

7 Ce faisant, reformulant le préambule de la loi d'orientation de 1989 qui affirme que l'élève est ou doit être « au centre du système éducatif $»$.
8 La méthode Boscher (datant de 1954) a par exemple été rééditée sans aucune modification en 1984 et demeure utilisée dans certaines classes.

9 «La main à la pâte » est un dispositif lancé en 1996 à l'initiative de Georges Charpak, prix Nobel de physique, et avec le soutien de l'Académie des sciences et de l'Institut français de l'Éducation, visant à rénover l'enseignement des sciences et de la technologie à l'école primaire, en favorisant un enseignement fondé sur une démarche d'investigation scientifique. II reprend ou propose des principes et des démarches similaires ou proches des démarches prônées par Freinet et recommandées quand existaient les enseignements dits d'éveil dans les années quatre-vingt.

10 Même si on peut lire, dans les travaux de didactique, beaucoup de comparaisons entre une ou deux classes de ZEP et une ou deux classes hors ZEP, et des études portant sur les modalités d'enseignement en ZEP, ou encore dans des classes faibles (Bautier, 2002a, 2002b ; Butlen, Peltier-Barbier \& Pézard, 2002 ; Peltier-Barbier, 2004 ; Perrin-Glorian, 1993, 1997).

11 Dans ce même numéro, voir l'entretien d'Anne Armand qui souligne également ce qu'elle nomme une " pédagogie de la lenteur".

12 Sur ces processus de catégorisation des élèves en dificulté, voir entre autres Monfroy (2002).

13 On pourrait tenir le même raisonnement à propos de l'étendue des connaissances encyclopédiques des élèves.

\section{BIBLIOGRAPHIE}

ARMAND A. \& GILLE B. (2006). La contribution de l'éducation prioritaire à l'égalité des chances des élèves. Rapport IGEN et IGAENR no 2006-076 au ministre de l'Éducation nationale, de l'Enseignement supérieur et de la Recherche. Paris : ministère de l'Éducation nationale, de l'Enseignement supérieur et de la Recherche.

BACHMANN C. (1977). " "Il les a dit devant lui et il n'avait pas peur", analyse de conversation ». Pratiques, n० 17, p. 75-99.

BACHMANN C., DURO-COURDESSES L., CHAUVEAU G. et al. (1983). Les zones d'éducation prioritaire. Actes de l'université d'été du 4 au 20 juillet 1983. Villetaneuse : Université Paris-Nord.

BAUTIER É. (2002a). «Du rapport au langage : question d'apprentissages différenciés ou de didactique ? " Pratiques, no 113-114, p. 41-54.

BAUTIER É. (2002b). " L'enseignement en ZEP et les recherches en didactique du français ". Revue française de pédagogie, no 140, p. 53-64.

BAUTIER É. (2005). Apprendre à l'école, apprendre l'école. Des risques de construction de l'inégalité dès la maternelle. Lyon : Éd. La Chronique sociale.

BERNSTEIN B. (1975). Langage et classes sociales. Paris : Éd. de Minuit.

BUTLEN D., PELTIER-BARBIER M.-L. \& PÉZARD M. (2002). «Nommés en REP, comment font-ils ? Pratiques de professeurs d'école enseignant les mathématiques en REP. Contradictions et cohérence ». Revue française de pédagogie, no 140, p. 41-52.
CENTRE DE RECHERCHE DE L'ÉDUCATION SPÉCIALISÉE ET DE L'ADAPTATION SCOLAIRE (CRESAS) (1981). Le handicap socio-culturel en question. Paris : ESF.

CHAUVEAU G. (2000). Comment réussir en ZEP ? Vers des zones d'excellence pédagogique. Paris : Retz.

FRANDJI D. \& ROCHEX J.-Y. (2011). « De la lutte contre les inégalités à l'adaptation aux besoins spécifiques". Éducation et formations, n० 80, p. 95-108.

HALTÉ J.-F. (1982a). « Aprendre autrement à l'école ». Pratiques, n० 36, p. 5-23.

HALTÉ J.-F. (1982b). « Travailler en projet ». Pratiques, no 36, p. 37-77.

HALTÉ J.-F. (1992). La didactique du français. Paris : PUF.

ISAMBERT-JAMATI V. (1985). «Quelques rappels de l'émergence de l'échec scolaire comme problème social dans les milieux pédagogiques français ". In É. Plaisance (dir.), L'"échec scolaire ", nouveaux enjeux, nouvelles approches sociologiques. Paris : Éd. du CNRS, p. 155163.

JOIGNEAUX C. (2009a). Des processus de différenciation dès l'école maternelle. Historicités plurielles et inégalité scolaire. Thèse de doctorat, sciences de l'éducation, université Paris 8-Vincennes-Saint-Denis.

JOIGNEAUX C. (2009b). « La construction de l'inégalité scolaire dès l'école maternelle ". Revue française de pédagogie, no 169, p. 17-28.

KHERROUBI M. \& ROCHEX J.-Y. (2002). « La recherche en éducation et les ZEP en France. Première partie : politique ZEP, objets, postures et orientations de 
recherche ". Revue française de pédagogie, no 140, p. $103-131$.

KHERROUBI M. \& ROCHEX J.-Y. (2004). « La recherche en éducation et les ZEP en France. Deuxième partie : apprentissage et exercice professionnel en ZEP : résultats, analyses, interprétations ". Revue française de pédagogie, no 146, p. 115-190.

LAHIRE B. (1993). Culture écrite et inégalité scolaire. Lyon : Presses universitaires de Lyon.

LAPARRA M. (1991). «Lire les livres d'histoire ». Pratiques, n० 69, p. 97-124.

LAPARRA M. (2006). « La grande section de maternelle et la raison graphique ». Pratiques, no 131-132, p. 237-249.

LAPARRA M. \& MARGOLINAS C. (2010). « Milieu, connaissance, savoir. Des concepts pour l'analyse des situations d'enseignement ». Pratiques, no 145-146, p. 141160.

MINISTÈRE DE L'ÉDUCATION NATIONALE (1998). «Orientations pédagogiques pour les enseignements généraux et professionnels adaptés dans le second degré ». Circulaire no 98-129 du 19 juin 1998. Bulletin officiel de l'Éducation nationale, 25 juin 1998, n० 26.

MINISTÈRE DE L'ÉDUCATION NATIONALE (2003). La discrimination positive en France et dans le monde. Actes du colloque international organisé les 5 et 6 mars 2002 à Paris. Paris : CNDP.

MOISAN C. \& SIMON J. (1997). Les déterminants de la réussite scolaire en zone d'éducation prioritaire. Rapport IGEN et IGAEN au ministre de l'Éducation nationale. Paris : La Documentation française.

MONFROY B. (2002). " La définition des élèves en difficulté en ZEP : le discours des enseignants de l'école primaire ". Revue française de pédagogie, no 140, p. 33-40.

NONNON É. (2010). "La notion de progression au cœur des tensions dans l'activité d'enseignement ". Repères, n० 41, p. 5-34.
PELTIER-BARBIER M.-L. (2004). Dur d'enseigner en ZEP. Grenoble : Éd. La Pensée sauvage.

PERRIN-GLORIAN M.-J. (1993). «Questions didactiques soulevées à partir de l'enseignement des mathématiques dans les classes "faibles" ". Recherches en didactique des mathématiques, vol. 13, no 1-2, p. 95-118.

PERRIN-GLORIAN M.-J. (1997). «Que nous apprennent les élèves en difficulté en mathématiques ? " Repères IREM, n०29, p. 43-66.

Pratiques (1999), no 101-102 : « Textes officiels et enseignement du français ».

PRIVAT J.-M. (2010). « Un bain de littératie. À l'école de la piscine 》. Ethnographiques.org, no 20. En ligne : http://www.ethnographiques.org/2010/Privat (consulté le 2 décembre 2011).

RAVON B. (2000). L'échec scolaire. Histoire d'un problème public. Paris : Éd. In Press.

ROCHEX J.-Y. (2008). «Vingt-cinq ans de politique d'éducation prioritaire en France : une spécificité incertaine et des résultats décevants ". In M. Demeuse, D. Frandji, D. Greger \& J.-Y. Rochex (dir.), Les politiques d'éducation prioritaire en Europe. Vol. 1 : Conceptions, mises en œuvre, débats. Lyon : INRP.

ROCHEX J.-Y. (2010). " Les trois "âges" des politiques d'éducation prioritaire : une convergence européenne ? » In C. Ben Ayed (dir.), L'école démocratique. Vers un renoncement politique? Paris : Armand Colin.

ROCHEX J.-Y. \& CRINON J. (2011). La construction des inégalités scolaires. Au cœur des pratiques et des dispositifs d'enseignement. Rennes : Presses universitaires de Rennes.

WEBER E. (1983). La fin des terroirs. La modernisation de la France rurale, 1870-1914. Paris : Fayard. 